\title{
DISTRIBUIÇÃO DE SELÊNIO EM ORGANISMOS MARINHOS DA BAÍA DE GUANABARA/ RJ
}

\author{
Tércia Guedes Seixas* e Isabel Moreira \\ Departamento de Química, Pontifícia Universidade Católica - Rio, Rua Marquês de São Vicente, 225,
} 22453-900 Rio de Janeiro - RJ, Brasil

\section{Helena do Amaral Kehrig e Olaf Malm}

Instituto de Biofísica Carlos Chagas Filho, Centro de Ciências da Saúde, Universidade Federal do Rio de Janeiro, 21949-900 Rio de Janeiro - RJ, Brasil

Recebido em 1/2/06; aceito em 3/7/06; publicado na web em 19/1/07

\begin{abstract}
DISTRIBUTION OF SELENIUM IN MARINE ORGANISMS FROM GUANABARA BAY/ RJ. The study assessed selenium concentrations in the muscle tissues and liver samples of three fish species and in the soft tissues of a mussel species. The samples were analyzed by GF-AAS using Zeeman background correction. Selenium in the muscle samples was higher in carnivorous fish. The liver of all studied fish species presented higher selenium concentrations than the muscle. The fish species presented a high positive correlation between their total length and selenium concentration. A significant correlation was observed between the selenium concentration in the mussel soft tissues and the condition index.
\end{abstract}

Keywords: total selenium; fish and mussel; Guanabara Bay.

\section{INTRODUÇÃO}

O selênio (Se) é conhecido como um micronutriente essencial para a maioria dos animais, porém em concentrações elevadas é considerado tóxico ${ }^{1,2}$. Estudos vêm mostrando que a morte de algumas espécies de organismos mais susceptíveis ao selênio é observada em ambientes aquáticos cuja água apresentou concentração de Se na faixa de 0,06 a $0,6 \mu \mathrm{g} \mathrm{g}^{-12}$. Para o ser humano, o Se é considerado como essencial em concentrações que variam de 0,04 a $0,1 \mu \mathrm{g} \mathrm{g}^{-12}$. Entretanto, para os organismos marinhos, como os peixes, o selênio se torna tóxico a partir de $3,0 \mu \mathrm{g} \mathrm{g}^{-1}{ }^{3}$. Há um grande interesse em se entender o comportamento do Se nos organismos marinhos, devido a sua importância fisiológica e toxicológica. Existem poucos trabalhos dedicados ao metabolismo e à acumulação do Se no meio ambiente aquático e seu ciclo biogeoquímico é parcialmente conhecido ${ }^{4}$. Alguns estudos relacionados ao metabolismo do Se nos produtores primários ${ }^{5,6}$ e acumulação do Se pelos consumidores ${ }^{7}$ forneceram algumas informações básicas a respeito da importância e do papel deste elemento para a biota marinha. Os bivalvos e os peixes são reconhecidamente empregados como biomonitores de Se em ambientes aquáticos ${ }^{1}$. Peters e colaboradores ${ }^{8}$ concluíram que a cadeia alimentar bentônica é uma importante fonte de Se para os peixes e que a principal rota para a bioacumulação é via cadeia alimentar. Porém, uma variedade de parâmetros abióticos e bióticos pode influir na eficiência com que os organismos aquáticos acumulam os metais pesados e o selênio em seus tecidos e órgãos ${ }^{9}$. A concentração de Se nos detritos orgânicos dos sedimentos é mais importante para a contaminação da cadeia alimentar aquática que a concentração de Se dissolvido na coluna d'água ${ }^{8}$. Estudos vêm mostrando que a contaminação dos sistemas aquáticos por Se pode causar sérios danos à biota, inclusive a mortalidade de peixes ${ }^{10}$.

Neste trabalho foram determinadas as concentrações de selênio total em tecido muscular e fígado de três espécies de peixes bentônicos com diferentes hábitos alimentares (Mugil liza - tainha, peixe

*e-mail: tercia@rdc.puc-rio.br planctívoro; Bagre spp. - bagre, peixe onívoro e Micropogonias furnieri - corvina, peixe carnívoro) e nos tecidos moles de uma espécie de mexilhão (Perna perna), coletados na Baía de Guanabara.

A Baía de Guanabara $\left(22^{\circ} \mathrm{S}, 43^{\circ} \mathrm{W}\right)$ é um estuário de aproximadamente $400 \mathrm{~km}^{2}$, localizada no estado do Rio de Janeiro, na região sudeste brasileira (Figura 1). É uma importante área com relação à produção pesqueira, porém recebe impactos na forma de esgotos domésticos e efluentes industriais não tratados, que são provenientes de uma área densamente povoada, com aproximadamente 10.000 indústrias, além de uma intensa atividade portuária e um complexo pólo petroquímico ${ }^{11}$. Sua bacia de drenagem pode ser considerada como uma região bastante impactada por matéria orgânica, óleo e um grande número de outros compostos, incluin-

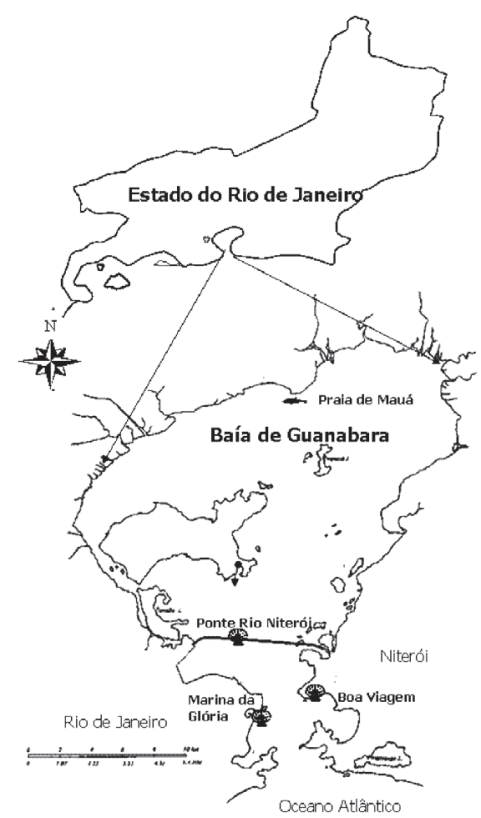

Figura 1. Locais de coleta na Baía de Guanabara, estado do Rio de Janeiro 
do-se os metais pesados ${ }^{12}$. Como consequiência, encontram-se elevadas concentrações de metais tóxicos e hidrocarbonetos nos sedimentos e alterações nas comunidades bentônicas e pelágicas ${ }^{11,13}$. A Baía de Guanabara tem sido objeto de vários estudos ambientais, porém, segundo a literatura científica, não são encontrados dados a respeito de selênio, tanto no ambiente quanto na biota deste importante estuário brasileiro.

\section{PARTE EXPERIMENTAL}

\section{Coleta e preparo das amostras}

Durante os anos de 2000 e 2003, foram coletadas na Baía de Guanabara, com auxílio da colônia de pesca da Praia de Mauá (município de Magé, RJ) (Figura 1), 31 indivíduos de tainha (peixe planctívoro), 14 indivíduos de bagre (peixe onívoro), 34 indivíduos de corvina (peixe carnívoro), perfazendo um total de 79 indivíduos. Após cada coleta, foram tomados o peso e o comprimento total de cada peixe. Em seguida, foram retirados o músculo dorso lateral esquerdo e o fígado de cada indivíduo. Também foram coletados, manualmente, com auxílio de estiletes 40 indivíduos de mexilhão (bivalvo filtrador) em três pontos distintos da baía: Marina da Glória $(\mathrm{N}=10)$, Praia da Boa Viagem $(\mathrm{N}=10)$ e Ponte Rio-Niterói $(\mathrm{N}=20)$ (Figura 1). Em seguida, foram tomados os comprimentos da concha e foram pesados os tecidos moles de cada indivíduo. Posteriormente, fez-se uma amostra composta homogênea de aproximadamente 10 indivíduos por amostra. As amostras de tecido muscular dos peixes e as amostras de tecidos moles dos mexilhões foram liofilizadas perdendo, aproximadamente, 75 e $80 \%$ de água, respectivamente.

\section{Instrumental}

A determinação de selênio total nas amostras foi realizada através de espectrofotômetro de absorção atômica da Analytik Jena AG (Jena, Alemanha), modelo ZEEnit 60, equipado com um corretor Zeeman de "background" e um auto amostrador MPE-52 (Analytik Jena AG). Como célula de absorção foi utilizado um forno de grafite (pirolítico) com aquecimento transversal e plataforma PIN (Analytik Jena AG, Part $N^{\circ}$ 407-152.011). Como fonte de radiação foi utilizada uma lâmpada de catodo oco de selênio (Analytik Jena AG, Part $\mathrm{N}^{\circ}$ 226832), operando a 6,0 mA, no comprimento de onda de 196,0 nm, com uma fenda de 1,2 nm. Argônio (95\% de pureza) foi utilizado com gás inerte de carreamento e proteção do forno de grafite. As leituras foram realizadas por área do pico de absorvância, utilizando um tempo de integração de $5 \mathrm{~s}$, sendo feitas em duplicata e descontados os respectivos brancos. As etapas do programa de temperatura empregado são apresentadas na Tabela 1.

Tabela 1. Programa de temperatura empregado para determinação de Se-T em amostras biológicas

\begin{tabular}{lcccc}
\hline Etapa & $\begin{array}{c}\text { Temperatura } \\
\left({ }^{\circ} \mathrm{C}\right)\end{array}$ & $\begin{array}{c}\text { Rampa } \\
\left({ }^{\circ} \mathrm{C} \mathrm{s}{ }^{-1}\right)\end{array}$ & $\begin{array}{c}\text { Sustentação } \\
(\mathrm{s})\end{array}$ & $\begin{array}{c}\text { Fluxo } \\
\text { de argônio }\end{array}$ \\
\hline $1^{\text {a }}$ secagem & 90 & 10 & 15 & máximo \\
$2^{\text {a }}$ secagem & 120 & 15 & 25 & máximo \\
Pré-pirólise & 500 & 7 & 20 & máximo \\
Pirólise & 1200 & 30 & 30 & máximo \\
Auto Zero & 1200 & 0 & 6 & zero \\
Atomização & 2200 & 2600 & 6 & zero \\
Limpeza & 2500 & 1000 & 5 & médio \\
\hline
\end{tabular}

\section{Soluções e reagentes}

Todos os reagentes utilizados foram de grau analítico e não foram previamente purificados. Ácido nítrico suprapuro (65\% p/v) (Merck) foi utilizado tanto na digestão das amostras, quanto no preparo das soluções estoque. Água de alta pureza (Sistema Millipore Milli-Q) foi usada em todas as diluições. Uma solução estoque de $1.000 \mu \mathrm{g} \mathrm{mL}^{-1}$ de $\mathrm{SeO}_{2}$ (Titrisol ${ }^{\oplus}$, Merck) foi preparada pela dissolução com uma solução $0,2 \%$ v/v de $\mathrm{HNO}_{3}$. As soluções de trabalho $\left(50 \mathrm{ng} \mathrm{mL}^{-1}\right)$ de selênio foram obtidas a partir da diluição da solução estoque de $1.000 \mu \mathrm{g} \mathrm{mL}^{-1}$ com uma solução $0,2 \%$ v/ v de $\mathrm{HNO}_{3}$ (Merck), e foram preparadas diariamente. Uma solução $1,5 \mu \mathrm{g} \mathrm{mL}^{-1}$ de nitrato de paládio (Merck) foi utilizada como modificador químico. Foi preparada a partir da diluição, em água deionizada, de uma solução estoque de $10.000 \mu \mathrm{g} \mathrm{mL}^{-1}$ de $\mathrm{Pd}\left(\mathrm{NO}_{3}\right)_{2}$ (Merck).

\section{Procedimento analítico}

A metodologia analítica aplicada neste estudo baseou-se no trabalho de Deaker e Maher ${ }^{14}$. Neste estudo foram empregadas cerca de $0,1 \mathrm{~g}$ de amostra seca ou 0,2 $\mathrm{g}$ de amostra úmida, que sofreram digestão ácida em 2,0 $\mathrm{mL}$ de $\mathrm{HNO}_{3}$ suprapuro concentrado (65\%p/v) (Merck) por aproximadamente $2 \mathrm{~h}$ a $60{ }^{\circ} \mathrm{C}$ em banhomaria, utilizando tubos de Pirex ${ }^{\circledR}$ fechados. Após o resfriamento, a solução da amostra digerida foi diluída cinco vezes em água Milli$\mathrm{Q}^{\circledR}$ (Sistema Millipore) e uma alíquota de $20 \mu \mathrm{L}$ foi retirada e analisada pela técnica do forno de grafite. Utilizou-se sempre uma curva analítica de selênio inorgânico $\left(\mathrm{SeO}_{2}\right)$ (Titrisol ${ }^{\circledR}$, Merck) em meio ácido, que precedeu a cada dia de análise.

O controle de qualidade analítica do método foi determinado utilizando-se, a cada batelada de análise, amostras de materiais certificados de referência fornecidos pelo "National Research Council Canada" (DORM-2, tecido muscular) e pelo "National Institute for Science and Technology" (EUA, NIST 2976, tecidos moles de mexilhão). As metodologias de digestão e de determinação de Se, descritas anteriormente, foram aplicadas a duplicatas de cada amostra. O coeficiente de variação (C.V.) encontrado para cada amostra foi sempre inferior a $\pm 10 \%$, significando que o procedimento analítico aplicado foi plenamente satisfatório.

\section{Análise estatística}

Após verificar-se a não normalidade na distribuição dos dados através do teste de Shapiro-Wilk (teste $\mathrm{W} ; \mathrm{p}=0,00$ ), aplicaram-se, os testes não paramétricos: análise de variância (Kruskal-Wallis ANOVA), para se testar as diferenças na concentração de Se entre as espécies, níveis tróficos, tecidos e hábito alimentar; e MannWhitney (Teste U) para se comparar a concentração de selênio nos diversos tecidos dos organismos. Também, utilizou-se a correlação de Spearman para verificar se havia relação entre os parâmetros estudados.

\section{RESULTADOS E DISCUSSÃO}

\section{Controle de qualidade analítica}

A precisão e exatidão do método analítico foram verificadas através da utilização de materiais certificados de referência fornecidos pelo "National Research Council Canada" (DORM-2, tecido muscular) e pelo "National Institute for Science and Technology" (EUA, NIST 2976, tecidos moles de mexilhão) e também através de uma comparação interlaboratorial entre o laboratório de refe- 
rência do "Finish National Public Health Institute" (FNPHI) e o Laboratório de Radioisótopos Eduardo Penna Franca (LREPF UFRJ) utilizando amostras de tecido muscular e fígado de peixes $(\mathrm{N}=9)$. Os resultados das análises das amostras de materiais de referência são apresentados na Tabela 2. Na Figura 2 são apresentados os resultados das análises da comparação interlaboratorial realizada entre os dois laboratórios.

Tabela 2. Concentração de Se-T nos materiais de referência empregados

\begin{tabular}{lcccc}
\hline Amostra & $\begin{array}{c}\text { Valor Certificado } \\
\text { Média } \pm \text { d.p. } \\
\text { intervalo }(95 \%)\end{array}$ & $\begin{array}{c}\text { Valor Obtido } \\
\text { Média } \pm \text { d.p. } \\
\text { intervalo (95 \%) }\end{array}$ & $\mathrm{N}$ & $\begin{array}{c}\text { Recuperação } \\
(\%)\end{array}$ \\
\hline $\begin{array}{l}\text { DORM-2 } \\
\left(\mu \mathrm{g} \mathrm{g}^{-1}\right)\end{array}$ & $1,40 \pm 0,09$ & $1,39 \pm 0,23$ & 26 & 99 \\
$\begin{array}{l}\text { NIST 2976 } \\
\left(\mu \mathrm{g} \mathrm{g}^{-1}\right)\end{array}$ & $1,80 \pm 0,15$ & $1,91 \pm 0,14$ & 10 & 101 \\
\hline
\end{tabular}

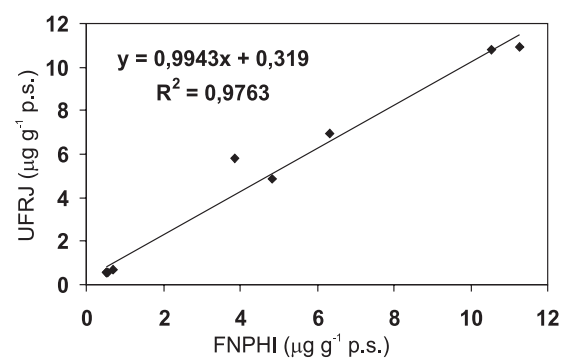

Figura 2. Resultado do exercício de calibração interlaboratorial realizado entre o LREPF e o FNPHI

Os resultados obtidos demonstraram a boa precisão e exatidão da metodologia analítica empregada. Obtiveram-se bons fatores de recuperação do Se-T, nas diferentes matrizes certificadas tanto do "National Research Council" (Canadá, DORM-2) quanto do "National Institute for Science and Technology" (EUA, NIST 2976) (Tabela 3). A comparação entre os dois laboratórios mostrou uma boa concordância dos dados, com uma correlação altamente significativa $\left(r^{2}=0,98\right)$, indicando que os resultados encontrados neste trabalho, no LREPF (UFRJ), foram tão precisos quanto os do FNPHI. O valor do coeficiente angular da equação $(a=0,99)$, que se apresentou próximo a 1 (tangente $45^{\circ}$ ), mostrou que os resulta- dos encontrados pelo Laboratório de Radioisótopos Eduardo Penna Franca foram tão exatos quanto os do laboratório de referência para análise de selênio do FNPHI. O teste $t$ mostrou que os resultados são similares, pois, o valor $t$ encontrado $(t=0,71)$ é muito menor que $t$ tabelado $(t=1,75)$ para $\mathrm{N}=9$ e $p=0,05$. Estes resultados mostram claramente, que o método analítico empregado, desde a digestão das amostras até a determinação de selênio pela técnica do forno de grafite, mostrou-se eficiente e reprodutivo para a análise de selênio em amostras de organismos aquáticos.

O limite de detecção $(3 \sigma)$ para o método foi de $1,00 \mu \mathrm{g} \mathrm{L}^{-1}$ e o limite de quantificação (L.Q.) foi de $0,03 \mu \mathrm{g} \mathrm{g}^{-1}$.

\section{Biota aquática}

Os resultados das medianas das concentrações de selênio total determinadas nos tecidos e órgãos das espécies estudadas e seus respectivos hábitos alimentares são apresentados na Tabela 3 e Figura 3.

As concentrações de Se no tecido muscular dos peixes mostraram-se significativamente diferentes $\left(\mathrm{H}=31,90 ; \mathrm{p}<1 \times 10^{-4}\right)$ e foram mais elevadas na corvina, peixe bentônico carnívoro. Entretanto, esta espécie de peixe apresentou uma concentração de Se no fígado similar $(\mathrm{p}=0,88)$ à encontrada no mesmo órgão da tainha e significativamente diferente à do fígado do bagre $\left(\mathrm{H}=17,46 ; \mathrm{p}<1 \times 10^{-4}\right)$. Trabalhos preliminares verificaram a influência do hábito alimentar e da posição trófica na acumulação de selênio ${ }^{15-18}$. Todos observaram que as espécies carnívoras ou piscívoras, geralmente, apresentaram maiores concentrações de selênio no tecido muscular que as espécies onívoras ou planctívoras. As concentrações de selênio obtidas no presente estudo apresentaram-se menores e dentro de uma variação habitual quando comparadas às concentrações observadas em estudos anteriores em



Figura 3. Mediana das concentrações de selênio total (Se-T) nos tecidos (músculo, fígado e tecidos moles) dos organismos estudados (peixes e mexilhão)

Tabela 3. Mediana das concentrações de Se-T ( $\mu \mathrm{g} \mathrm{g}^{-1}$ p.u.) nos tecidos e órgãos dos organismos da Baía de Guanabara e seus parâmetros biológicos

\begin{tabular}{|c|c|c|c|c|}
\hline & $\begin{array}{c}\text { Micropogonias furnieri } \\
\text { (corvina) }\end{array}$ & $\begin{array}{l}\text { Bagre spp. } \\
\text { (bagre) }\end{array}$ & $\begin{array}{l}\text { Mugil liza } \\
\text { (tainha) }\end{array}$ & $\begin{array}{l}\text { Perna perna } \\
\text { (mexilhão) }\end{array}$ \\
\hline & $\begin{array}{c}\text { (N amostral) } \\
{[\text { Se-T }]}\end{array}$ & $\begin{array}{c}\text { (N amostral) } \\
{[\mathrm{Se}-\mathrm{T}]}\end{array}$ & $\begin{array}{c}\text { (N amostral) } \\
{[\text { Se-T }]}\end{array}$ & $\begin{array}{c}\text { (N amostral) } \\
{[\text { Se-T] }}\end{array}$ \\
\hline & (mín. - máx.) & (mín. - máx.) & (mín. - máx.) & (mín. - máx.) \\
\hline \multirow[t]{2}{*}{ Tecido muscular } & $(\mathrm{N}=34) 0,29$ & $(\mathrm{~N}=14) \quad 0,07$ & $(\mathrm{~N}=31) \quad 0,11$ & - \\
\hline & $(0,12-1,25)$ & $\left(<\mathrm{LQ}^{\mathrm{a}}-0,12\right)$ & $\left(<\mathrm{LQ}^{\mathrm{a}}-0,18\right)$ & \\
\hline \multirow[t]{2}{*}{ Fígado } & $(\mathrm{N}=14) 2,47$ & $(\mathrm{~N}=14) \mathbf{1 , 3 0}$ & $(N=13) 2,44$ & - \\
\hline & $(0,39-7,53)$ & $(1,17-1,71)$ & $(1,04-13,67)$ & \\
\hline Tecidos moles & - & - & - & $\begin{array}{c}(\mathbf{N}=\mathbf{4 0}) \mathbf{0 , 1 6} \\
(0,12-0,27)\end{array}$ \\
\hline Comprimento total médio (mm) & $390(340-580)$ & $400(320-540)$ & $360(290-500)$ & $63(45-80)$ \\
\hline Peso médio (g) & $750(360-1900)$ & $710(330-1700)$ & $560(310-1620)$ & $7,8(2,69-14,95)$ \\
\hline Hábito alimentar & carnívoro & onívoro & planctívoro & filtrador \\
\hline
\end{tabular}

${ }^{\mathrm{a}} \mathrm{LQ}=$ limite de quantificação. 
outros ecossistemas mundiais, como é mostrado na Tabela 4 e algumas vezes, abaixo daquelas relatadas em peixes de consumo humano em outros países (Tabela 5). O fígado dos peixes estudados foi o tecido que apresentou a maior concentração de Se quando comparado ao tecido muscular dos mesmos. Estas observações estão consistentes com estudos realizados com peixes de outros ecossistemas mundi-


estudo, para o fígado dos peixes apresentaram-se menores e dentro de uma variação habitual quando comparadas às concentrações em ambientes não impactados por selênio relatadas em estudos anteriores com peixes, como mostra a Tabela 6 .

Tabela 4. Intervalos das concentrações médias de $\mathrm{Se}-\mathrm{T}\left(\mu \mathrm{g} \mathrm{g} \mathrm{g}^{-1}\right)$ reportadas no tecido muscular de peixes em outros ecossistemas

\begin{tabular}{lc}
\hline Ecossistema, País & {$[\mathrm{Se}-\mathrm{T}] \mu \mathrm{g} \mathrm{g}^{-1}$} \\
\hline Estuário de Lake & $3,4^{\mathrm{b}}-9,3^{\mathrm{c}}$ (p.s.) \\
${\text { Macquarie, Austrália }{ }^{17,19-21}} 0,1-0,3$ (p.u.) \\
& $0,3-13$ (p.u.) \\
Raine Island, Austrália $^{22}$ & $0,33-3,47$ (p.u.) \\
Todo E.U.A. $^{23}$ & $0,39-0,80$ (p.u.) \\
${\text { Savannah River, E.U.A. }{ }^{16}}^{16}$ & $0,7-0,82$ (p.u.) \\
Sunderban delta, Índia & \\
& $0,21-0,64$ (p.u.) \\
Baía de Guanabara, Brasil & $1,27-1,96$ (p.s.) \\
\hline
\end{tabular}

(p.u.) concentração expressa em peso úmido; (p.s.) concentração

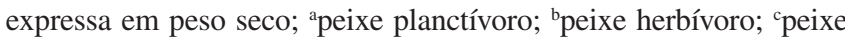
carnívoro; 'peixe onívoro; *variação dos valores encontrados neste estudo no tecido muscular dos peixes estudados.

Tabela 5. Concentração média de Se-T $\left(\mu \mathrm{g} \mathrm{g}^{-1}\right)$ relatada em peixes de consumo humano de diferentes países

\begin{tabular}{lc}
\hline País & $\begin{array}{c}\text { Concentração médiade Se-T } \\
\left(\mu \mathrm{g} \mathrm{g}^{-1} \text { p.u. }\right)\end{array}$ \\
\hline Austrália $^{24}$ & $1,0^{\mathrm{a}}$ \\
Bélgica $^{25}$ & 0,25 \\
Estados Unidos $^{25}$ & 0,53 \\
França $^{25}$ & 0,20 \\
Inglaterra $^{25}$ & 0,32 \\
Sonora, México $^{26}$ & 0,90 \\
Baía de Guanabara, Brasil & $0,07-0,29^{*}$ \\
\hline
\end{tabular}

${ }^{a}$ concentração máxima permitida no tecido muscular segundo a ref. 24; "variação dos valores encontrados neste estudo no tecido muscular dos peixes estudados.

Tabela 6. Concentrações naturais de Se-T relatadas no fígado de peixes

\begin{tabular}{lc}
\hline Espécie & {$[\mathrm{Se}-\mathrm{T}]\left(\mu \mathrm{g} \mathrm{g}^{-1}\right)$} \\
\hline Peixe marinho $^{29}$ & $2,6-6,6$ (p.s.) \\
Peixe marinho $^{30-32}$ & $0,6-5,0$ (p.u.) \\
Peixe marinho $^{31}$ & $5,0-30,0$ (p.u.) \\
Peixe marinho $^{33}$ & 0,6 (p.u.) \\
Peixe $^{27}$ & $2,72-11,23$ (p.s.) \\
Atum japonês $^{34}$ & $10,0-15,0$ (p.u.) \\
Black marlin & 35 \\
Corvina & $1,4-13,5$ (p.u.) \\
Bagre & $0,39-7,53^{*}$ (p.u.) \\
Tainha & $1,17-1,71^{*}$ (p.u.) \\
\hline
\end{tabular}

(p.u.) concentração expressa em peso úmido; (p.s.) concentração expressa em peso seco; "variação dos valores encontrados neste estudo no fígado dos peixes estudados.
O teste $\mathrm{H}$ de Kruskal-Wallis-ANOVA mostrou haver diferença significativa na concentração de Se entre os tecidos $(\mathrm{H}=95,87$; $\mathrm{p}<1 \times 10^{-4}$ ). Este teste também foi aplicado ao conjunto de todos os dados com o intuito de se verificar qual dentre os tecidos (músculo e fígado) sofre maior influência do hábito alimentar na acumulação de Se. Observou-se que o músculo foi o único tecido que apresentou uma diferença significativa $\left(\mathrm{H}=31,90 ; \mathrm{p}<1 \times 10^{-4}\right)$ nas concentrações de Se em função do hábito alimentar de cada espécie de peixe. Portanto, conclui-se que o fígado, provavelmente, é um órgão que pouco sofre a influência do hábito alimentar. A exposição do organismo ao selênio via água, sedimento e alimento parece ser o principal fator que influi na acumulação deste metalóide pela biota aquática. Outros fatores que também podem influir são a espécie de peixe, o hábito alimentar, a posição trófica, o comprimento, peso e idade ${ }^{15}$.

A mediana da concentração de Se obtida para os tecidos moles do mexilhão, $0,16 \mu \mathrm{g} \mathrm{g}^{-1}$ em peso úmido ou $0,71 \mu \mathrm{g} \mathrm{g}^{-1} \mathrm{em}$ peso seco (Tabela 7), foi similar àquelas encontradas por Baldwin e Maher ${ }^{28}$ $\left(0,7-1,8 \mu \mathrm{g} \mathrm{g}^{-1} \mathrm{em}\right.$ peso seco) e inferiores àquelas encontradas em moluscos bivalvos de ambientes relativamente não impactados da Austrália, que variavam de 1,3 a $11 \mu \mathrm{g} \mathrm{g}^{-1}$ em peso $\operatorname{seco}^{36} \mathrm{e}$, em ambientes da costa indiana ${ }^{1}\left(3,94 \mu \mathrm{g} \mathrm{g}^{-1}\right.$ em peso seco). Porém, quando comparada às concentrações de Se encontradas nos peixes (Figura 3), se apresenta inferior àquelas encontradas no peixe carnívoro. Este fato vem em desencontro ao observado em trabalhos anteriores. onde os bivalvos acumularam mais selênio nos seus tecidos que os peixes $^{1}$. Neste estudo, tanto nos peixes quanto nos mexilhões, as concentrações de Se encontradas nos tecidos apresentaram-se abaixo do limite recomendável pela OMS $\left(1,5 \mu \mathrm{g} \mathrm{g}^{-1} \mathrm{em}\right.$ peso úmido) para o consumo humano e, também, apresentaram-se menores e dentro de uma variação habitual quando comparadas às concentrações relatadas em estudos anteriores com bivalvos, em áreas não impactadas por selênio, como mostra a Tabela 7 .

Tabela 7. Concentrações naturais de Se-T relatadas nos tecidos moles de bivalves

\begin{tabular}{lc}
\hline Espécie & {$[\mathrm{Se}-\mathrm{T}]\left(\mu \mathrm{g} \mathrm{g}^{-1}\right)$} \\
\hline Molusco bivalvo & $0,1-0,9$ (p.u.) \\
Molusco bivalvo $^{31,32,39,40}$ & $1,3-9,9$ (p.s.) \\
Mexilhão, Mytilus edulis ${ }^{41}$ & $>5,0$ (p.s.) \\
Mexilhão, Mytilus galloprovincialis ${ }^{7}$ & 6,0 (p.u.) \\
Mexilhão, Perna perna & $0,16^{*}$ (p.u.)0,71* (p.s.) \\
\hline
\end{tabular}

(p.u.) concentração expressa em peso úmido; (p.s.) concentração expressa em peso seco; ${ }^{*}$ valores encontrados neste estudo nos tecidos moles dos mexilhões estudados.

Na Tabela 8 são apresentadas as correlações testadas entre o comprimento total (L) e a concentração de Se nos tecidos dos organismos estudados.

Foram encontradas correlações significativas $(\mathrm{p}<0,05)$ e positivas entre as concentrações de Se no tecido muscular da corvina e tainha e seus respectivos comprimentos totais (L) (Tabela 8 e Figura 4). Estes resultados mostraram-se perfeitamente coerentes, uma vez que com o aumento do comprimento, há o aumento da idade do peixe e, conseqüentemente, o aumento do tempo de exposição do organismo ao Se, através do ambiente em que vive e, também, do alimento. Outros autores já verificaram a influência do fator tamanho na acumulação do selênio ${ }^{20,42-44}$. Esses autores não encontraram relações significativas $(\mathrm{p}<0,05)$ entre as concentrações de selênio e o comprimento total dos organismos. Somente Mackay e colaboradores ${ }^{35}$ encontraram relações significativas entre as con- 
Tabela 8: Resultados das análises de regressão linear para as concentrações de Se-T nos tecidos úmidos dos organismos da Baía de Guanabara, o comprimento total (L) dos peixes e o índice de condição (IC) pelos mexilhões

\begin{tabular}{llll}
\hline Tecido & \multicolumn{1}{c}{ Espécie } & \multicolumn{2}{c}{ Equação de regressão } \\
\hline Tecido Muscular & M. furnieri $(\mathrm{N}=34)$ & {$[\mathrm{Se}-\mathrm{T}]=0,041 \mathrm{~L}-1,251$} & $\mathrm{R}=0,84 ; p<1 \times 10^{-4 * *}$ \\
& Bagre spp. $(\mathrm{N}=14)$ & {$[$ Se-T $]=0,046 \mathrm{~L}+0,0005$} & $\mathrm{R}=0,15 ; p=0,61 \mathrm{~ns}$ \\
M. liza $(\mathrm{N}=31)$ & {$[\mathrm{Se}-\mathrm{T}]=0,006 \mathrm{~L}-0,099$} & $\mathrm{R}=0,64 ; p=1 \times 10^{-4} * *$ \\
Fígado & M. furnieri $(\mathrm{N}=14)$ & {$[$ Se-T] $=0,242 \mathrm{~L}-5,304$} & $\mathrm{R}=0,28 ; p=0,34 \mathrm{~ns}$ \\
& Bagre spp. $(\mathrm{N}=14)$ & {$[$ Se-T] $=0,007 \mathrm{~L}+1,057$} & $\mathrm{R}=0,26 ; p=0,37 \mathrm{~ns}$ \\
M. liza $(\mathrm{N}=13)$ & {$[$ Se-T] $=-0,559 \mathrm{~L}+21,270$} & $\mathrm{R}=-0,29 ; p=0,33 \mathrm{~ns}$ \\
Tecidos Moles & P. perna $(\mathrm{N}=40)$ & {$[$ Se-T] $=2,25 \mathrm{IC}-0,079$} & $\mathrm{R}=0,91 ; p=5 \times 10^{-4} * *$
\end{tabular}

(ns) - correlação não significativa; $(*)$ - correlação significativa $(\mathrm{p} \leq 0,05) ;(* *)$ - correlação altamente significativa $(\mathrm{p}<<0,001)$; ${ }^{\text {aC }}$ = ((massa de tecidos moles $(\mathrm{g}))$ / (comprimento da concha $(\mathrm{mm}))$ ). O IC foi utilizado para normalizar os dados, uma vez que existia uma diferença grande de tamanho entre os indivíduos coletados na Ponte Rio-Niterói ${ }^{44}$.

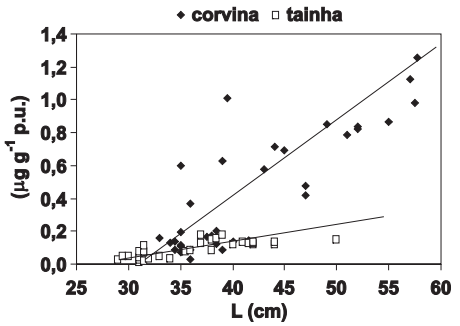

Figura 4. Representação gráfica da concentração de selênio total (Se-T) no tecido muscular com o comprimento total $(\mathrm{cm})$ da corvina e da tainha

centrações de selênio e o comprimento total, no tecido muscular e no fígado de peixes (marlim-negro).

Os mexilhões apresentaram uma correlação significativa e positiva entre a concentração de Se e o índice de condição (IC). O IC é um parâmetro utilizado na normalização de dados de acumulação de metais pesados em bivalvos ${ }^{45}$, pois, através deste pode-se excluir diferenças individuais, como tamanho, massa, estágio de vida, ciclo sexual, que são efeitos importantes no processo de bioacumulação de metais traço em mexilhões ${ }^{46-48}$. O teste $\mathrm{H}$ de Kruskal-Wallis-ANOVA mostrou haver diferença significativa na concentração de $\mathrm{Se}$, nos tecidos moles dos mexilhões, entre os diferentes locais de coleta na Baía de Guanabara (praia de Boa Viagem, Marina da Glória e Ponte Rio-Niterói) $(\mathrm{H}=5,96 ; \mathrm{p}=0,05)$. Estas observações vêm de encontro aos resultados observados com metais pesados no ecossistema da Baía de Guanabara. Kehrig e colaboradores $^{49}$ com o estudo de $\mathrm{Hg}$ total e metilmercúrio $(\mathrm{MeHg})$ e Lima $^{50}$ com $\mathrm{Cd}, \mathrm{Cr}, \mathrm{Cu}$ e $\mathrm{Zn}$ mostraram haver diferenças espaciais nas concentrações dos metais entre as áreas da Baía de Guanabara amostradas. Porém as concentrações de selênio foram semelhantes às encontradas em ambientes considerados não impactados ${ }^{1,28,36}$.

\section{CONCLUSÕES}

Apesar do ecossistema da Baía de Guanabara ser altamente impactado por substâncias tóxicas, provavelmente, este estuário não deva estar sofrendo lançamentos antropogênicos de selênio, uma vez que a biota analisada apresentou concentrações de selênio em seus tecidos semelhantes às de áreas consideradas não impactadas.

Verificou-se que o organismo predador foi o que apresentou as maiores concentrações de selênio no tecido muscular indicando, assim, que o hábito alimentar influi diretamente na acumulação de selênio neste tecido.

Há uma tendência do Se em se acumular em órgãos com atividade metabólica muito intensa (fígado) e esta pode não ser influenciada pelo hábito alimentar, mas sim pelo modo de incorporação do Se (via dieta ou água).

\section{AGRADECIMENTOS}

Ao suporte financeiro concedido pela CAPES através de uma bolsa de mestrado, CNPq (processo 476735/2003-3) e FAPERJ (processo E-26/170.998/2002).

\section{REFERÊNCIAS}

1. Chatterjee, A.; Bhattacharya, B.; Adv. Environ. Res. 2001, 5, 167.

2. Eisler, R.; U.S. Fish and Wildlife Service Biological Report 85 (1.5), 1985.

3. Lemly, A. D.; Ecotoxicol. Environ. Saf. 1997, 37, 259.

4. Pelletier, E.; Mar. Environ. Res. 1985, 18, 111.

5. Fries, L.; J. Phycology 1982, 18, 328.

6. Price, N. M.; Thompson, P. A.; Harrison, P. J.; J. Phycology 1987, 23, 1.

7. Fowler, S. W.; Benayoun, G.; Bull. Environ. Contam. Toxicol.1976, 16, 339.

8. Peters, G. M.; Maher, W. A.; Krikova, F.; Roach, A. C.; Jeswani, H. K.; Barford, J. P.; Gomes, V. G.; Reible, D. D.; Mar. Environ. Res. 1999, 47, 491.

9. Reindfelder, J. R.; Fisher, N. S.; Luoma, S. N.; Nichols, J. W.; Wang, W. X.; Sci. Total Environ. 1998, 219, 117.

10. Gillespie, R. B.; Baumann, P. C.; Trans. Am. Fisheries Soc. 1986, 115, 208.

11. Fundação Estadual de Engenharia do Meio Ambiente - RJ; Projeto de recuperação gradual do ecossistema da Baía de Guanabara, Rio de Janeiro, 1990

12. Rebello, A. L.; Haekel, W.; Moreira, I., Santelli, R.; Schroeder, F.; Mar. Chem. 1986, 18, 215.

13. Carreira, R. S.; Wagener, A. L. R.; Readman, J. W.; Fileman, T. W.; Macko, S. A.; Veiga, A.; Mar. Chem. 2002, 79, 202.

14. Deaker, M.; Maher, W.; Anal. Chim. Acta 1997, 350, 287.

15. Besser, J. M.; Giesy, J. P.; Brown, R. W.; Buell, J. M.; Dawson, G. A.; Ecotoxicol. Environ. Saf. 1996, 35, 7.

16. Burger, J.; Gaines, K. F.; Boring, C. S.; Stephens, W. L. Jr.; Snodgrass, J.; Gochfeld, M.; Environ. Res., A 2001, 87, 108.

17. Barwick, M.; Maher, W.; Mar. Environ. Res. 2003, 56, 471.

18. Bhattacharya, B.; Sarkar, S. K.; Das, R.; Ecological Indicators 2003, 2, 367.

19. Maher, W.; Baldwin, S.; Deaker, M.; Irving, M.; Appl. Organomet. Chem. 1992, 6, 103.

20. Roberts, B.; Unpublished BSc. Honorous Thesis, University of Canberra, 1993.

21. Wlodarczkyk, J.; Beath, K.; Heavy metals in seafood in Lake Macquarie: a cross-sectional survey, (Report to the Hunter Public Health Unit), University of Newcastle Research Association, New Lambton, New South Wales, 1997.

22. Rayment, G. E.; Barry, G. A.; Mar. Pollut. Bull. 2000, 41, 353.

23. Schmitt, C. J.; Brumbaugh, W. G.; Arch. Environ. Contam. Toxicol.1990, 19,731 .

24. Rayment, G.; Barry, G. A.; Mar. Pollut. Bull. 2000, 41, 353.

25. Simonoff, M.; Hamon, C.; Moreto, P.; Llabador, Y.; Simonoff, G.; J. Food Comp. Anal. 1988, 1, 295.

26. Wyatt, C. J.; Meléndez, J. M.; Acuña, N.; Rascon, A.; Nutr. Res. 1996, 16, 949.

27. Liu, D. L.; Yang, Y. P.; Hu, M. H.; Harrison, P. J.; Price, N. M.; Mar Environ. Res. 1987, 22, 151.

28. Baldwin, S.; Maher, W.; Mar. Environ. Res. 1997, 44, 243.

29. Grimanis, A. P.; Zafiropoulos, D.; Vassilaki-Grimani, M.; Environ. Sci. Technol. 1978, 12, 723. 
30. De Goeij, J. J. M.; Guinn, V. P.; Young, D. R.; Mearns, A. J.; Comparative studies of food and environmental contamination, International Atomic Energy Agency: Vienna, 1974, p. 189-200.

31. Hall, R. A.; Zook, E. G.; Meaburn, G. M.; National Marine Fisheries Service survey of trace elements in the fishery resource, U.S. Dep. Commer. NOAA Tech. Rep., NMFS SSRF-721, 1978.

32. Luten, J. B.; Ruiter, A.; Ritskes, T. M.; Rauchbaar, A. B.; Riekwel-Booy, G.; J. Food Sci. 1980, 45, 416.

33. Heit, M.; Bull. Environ. Contam. Toxicol. 1979, 23, 1.

34. Tamura, Y.; Maki, T.; Yamada, H.; Shimamura, Y.; Ochiai, S.; Nishigaki, S.; Kimura, Y.; Tokyo Toritsu Eisei Kenkyusho Nenpo 1975, 26, 200.

35. Mackay, N. J.; Kazacos, M. N.; Williamns, R. J.; Leedow, M. I.; Mar. Pollut. Bull. 1975, 6, 57.

36. Lobel, P. B.; Logerich, H. P.; Jackson, S. E.; Belkhode, S. P.; Arch. Environ Cont. Toxicol. 1991, 21, 118 .

37. Lunde, G.; J. Sci. Food Agric. 1970, 21, 242.

38. Bertine, K. K.; Goldberg, E. D.; Limnol. Ocean. 1972, 17, 877.
39. Karbe, L.; Schnier, Ch.; Slewers, H. O.; J. Radioanal. Chem.1977, 37, 927. 40. Fukai, R.; Oregioni, B.; Vas, D.; Oceanologica Acta 1978, 1, 391.

41. Stump, I. G.; Kearney, J. M.; D’Auria, J.; Popham, J. D.; Mar. Pollut. Bull. 1979, 10, 270.

42. Lyle, J. M.; Aust. J. Mar. Freshwat. Res. 1986, 37, 309.

43. Chvojka, R.; Mar. Pollut. Bull. 1988, 19, 210.

44. Maher, W.; Deaker, M.; Jolley, D.; Krikowa, B.; Roberts, B.; Appl. Organomet. Chem. 1997, 11, 313.

45. Joiris, C. R.; Holsbeek, L.; Otchere, F.A.; Mar. Pollut. 2000, 40, 457.

46. Wang, W.; Environ. Int. 1987, 13, 437.

47. Cossa, D.; Oceanologica Acta 1989, 12, 417.

48. Rebelo, M. F.; do Amaral, M. C. R.; Pfeiffer, W. C.; Mar. Pollut. Bull. 2003, 46, 1341.

49. Kehrig, H. A.; Costa, M.; Moreira, I.; Malm, O.; Mar. Pollut. Bull. 2002, 44, 1018.

50. Lima, E. F. A.; Dissertação de Mestrado, Pontifícia Universidade Católica, Brasil, 1997. 